\title{
Evoked skin sympathetic nerve responses in man
}

\author{
PAUL M SATCHELL, CHRISTOPHER P SEERS \\ From the Sobell Department of Neurophysiology, Institute of Neurology, London, UK
}

SUMMARY Activity in human unmyelinated efferent nerve fibres was recorded from seven upper limb cutaneous nerve fascicles. The activity induced by contralateral nerve trunk stimulation or tone burst was averaged and could be compared providing the stimuli were delivered at random times and in a random sequence. The average evoked sympathetic nerve responses to nerve trunk stimulation and tone burst were identical in latency and duration.

During the last decade microneuronography, a technique for recording from single and small numbers of myelinated and unmyelinated nerve fibres in conscious man, has been used to determine the characteristics of activity in sympathetic post-ganglionic nerve fibres innervating skin structures. This nerve activity consists of vasoconstrictor and sudomotor impulses involved in thermoregulation. ${ }^{1-4}$ One of the most prominent characteristics of skin sympathetic nerve activity is the ease with which a burst of impulses can be produced by an arousal stimulus. The dependence of skin sympathetic nerve activity on the state of alertness of the subject is so strong that sudden noise and mental stress are routinely used in the characterisation of nerve activity recorded microneuronographically. Indeed, this stimulus-response relationship has often provided the evidence that a recording comes from a new fascicle that predominantly innervates skin. In microneuronographic studies the arousal response has also been used in the characterisation of the relationships between nerve activity and skin effector organs, ${ }^{3}$ the determination of sympathetic reflex latencies, ${ }^{5}$ the changes in skin sympathetic nerve activity in spinal man, ${ }^{6}$ and in ascertaining the afferent or efferent nature of single unmyelinated fibres. ${ }^{7}$

Although the skin sympathetic responses to arousal stimuli have been used widely, they have also been described as being ephemeral, prone to adaptation and dominated by the novelty of any stimulus situation. ${ }^{1}$ This variation in the sympathetic responses to arousal has not interfered with the determination of

Address for reprint requests: Dr Paul Satchell, Gordon Craig Laboratory, Department of Surgery, University of Sydney, Sydney, Australia 2006.

Received 7 November 1986. Accepted 27 January 1987 the average latency of the skin sympathetic response to an electrical shock. ${ }^{5}$ These latencies can vary considerably but once averaged are virtually identical between different recordings in the same individual. There has been no attempt to determine any other consistent properties of the skin sympathetic nerve responses to electric stimuli nor have these responses been systematically compared with skin sympathetic responses produced by a variety of other stimuli. The proposition that the skin sympathetic responses to noise, mental stress and respiratory movement are identical to the responses from electrical stimulation has not been systematically examined. ${ }^{1}$

The aims of the present study were to determine the characteristics of evoked skin sympathetic nerve responses using averaging techniques and to test the hypothesis that the skin sympathetic nerve responses to nerve trunk stimulation and noise are identical.

\section{Methods}

One of us (PS, aged 36-37 years) was both the microneuronographer and subject in all 12 recording sessions. The skin fascicles were in the left ulnar nerve five times, left median nerve at the elbow and right median nerve at the wrist twice and in the right ulnar, left median nerve at the wrist and the right median nerve at the elbow. All ulnar fascicles were recorded at the wrist. Skin sympathetic nerve recordings that were stable for longer than an hour were obtained on seven occasions.

\section{Recording equipment}

Insulated tungsten electrodes, with uninsulated tips were used for nerve recording. A reference electrode, a used recording needle that had been sterilised, was inserted subcutaneously $2-3 \mathrm{~cm}$ from the recording site. The electrodes were connected to a differential preamplifier ${ }^{8}$ which had a gain of 1000 . This was connected via an optical isolator unit to an AA6Mk3 Biological Amplifier in a Medelec MS6 System (Filter settings $800-3200 \mathrm{~Hz}$ ). An amplitude discrimi- 
nator further improved the signal to noise ratio and the fullwave rectified signal was passed through a leaky integrator (time constant, $\mathbf{0 . 1 \mathrm { s } )}$ to produce a mean voltage display of the nerve signal. Nerve activity was monitored with a loud speaker and storage oscilloscope (Tetronix 5113). Nerve activity and other variables were stored on a 4-channel tape recorder (Racal Store 4). The ECG and respiratory movements were also recorded. In some experiments skin resistance outside the innervation zone of the impaled fascicle was recorded ${ }^{9}$ and vasomotor responses were monitored by a photoelectric pulse plethysmograph.

\section{Nerve recordings}

The subject sat in a comfortable chair. A microelectrode was inserted and adjusted until a skin nerve fascicle was impaled as judged by the insertion parathesiae and the mechanoreceptive afferent impulses arising in response to a touch stimulus within the fascicular receptive field. Further adjustment of the electrode position often resulted in recording skin sympathetic nerve activity. The characteristics of this nerve activity were identical to those described in previous studies, which have established that this activity consists of impulses in sympathetic vasoconstrictor and sudomotor motor fibres. ${ }^{1-35}$

\section{Experimental procedure}

Once stable skin sympathetic nerve recordings were obtained a variety of experimental protocols were followed. In a few initial studies electrical stimuli were delivered to the contralateral nerve trunk at fixed interstimulus intervals. Nerve trunks were stimulated with an isolated stimulator (NS6) and stimulus levels were 2-3 times the sensory threshold. In the majority of studies the electric stimuli were delivered randomly in time, the subject being blindfolded and aurally isolated. In some studies the skin sympathetic responses to auditory tone bursts were recorded. Tone bursts were produced by a Digitimer 4030 (TTL pulses, $700 \mathrm{~Hz}$ for $250 \mathrm{~ms}$ ) and were delivered randomly in time to the blindfolded subject via headphones. The intensity of the tone burst was 96dBA per ear (Briiel \& Kjaer Audio Frequency Spectrometer). A comparison of the evoked sympathetic responses to different stimuli (skin shock $v$ tone burst) was only carried out when the different stimuli were delivered in the one recording session. Each type of stimulus was delivered randomly in time and the choice of stimulus was also random.

\section{Analysis}

Marker pulses were recorded when electrical stimuli were given. The polarity of the marker pulse was used to distinguish different stimuli. The mean voltage neurogram of the skin sympathetic nerve activity was fed into an averager (Datalab DL4000B) which was triggered by the marker pulse. The average of 16 responses was identical to the average of a large number of responses (80). Three seconds of neurogram was averaged after each marker pulse. The resolution was $0.7 \mathrm{~ms}$ per point.

\section{Results}

General characteristics

Spontaneous nerve activity was of sympathetic origin as it satisfied criteria detailed previously. ${ }^{12}$ It was not possible to carry out the procedures of local blockade, to prove the efferent nature of the nerve activity, and duplicate recordings, to verify that the conduction velocity of bursts was in the unmyelinated range, because of the constraints of the recording situation. However, the pattern and temporal profile of the nerve activity (fig 1) suggested that it was identical to the skin sympathetic nerve activity recorded by others. ${ }^{12}$

A variety of stimuli, namely peripheral nerve trunk stimulation, skin stimulation, respiratory movement and auditory tone bursts could produce bursts of skin sympathetic nerve activity. These responses were of variable duration, amplitude and pattern (fig 2). As observed by others, an important determinant of the pattern of the response is the frequency of stimuli. Interstimulus intervals less than the duration of the responses resulted in great variation in the pattern of the responses. In this situation, many stimuli were not followed by responses (fig 3). In contrast, virtually all stimuli, if given infrequently enough, were followed by responses (figs 1 and 3).

\section{Averaging of evoked skin sympathetic responses}

The constant latency from the stimulus to the onset of successive average responses suggested that it was permissible to average evoked sympathetic responses.
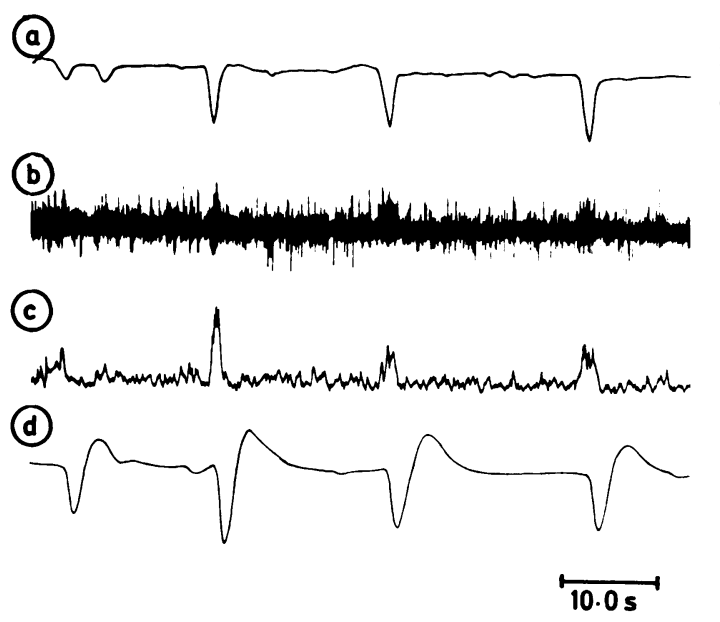

Fig 1 Sympathetic nerve activity recording in a fascicle of the left ulnar nerve innervating skin at the base of the left hypothenar eminence. Trace A: respiratory movement, inspiration downwards. Trace B: spontaneous multiunit nerve activity. Trace $C$ : integrated neural activity. Trace D: skin resistance recorded on palm proximal to the base of the little finger. Inspiratory movements were often followed by bursts of skin sympathetic activity, providing the respiratory rate was low. 


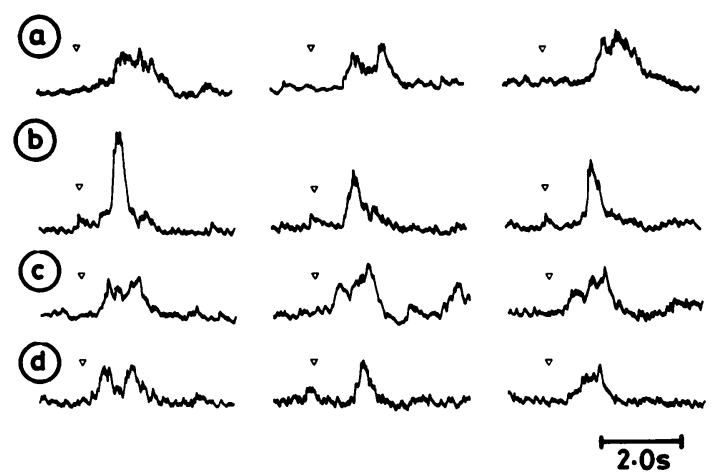

Fig 2 Three individual bursts of skin sympathetic nerve activity observed at four different recording sessions $(A, B, C, D)$ and displayed as integrated neural activity. The same stimulus was used in each recording session and the interval between the various stimuli was greater than 10 seconds. The onset of the stimulus is shown by the marker. (A) Recording in left ulnar nerve in response to respiration. (B) Recording in left ulnar nerve in response to stimulation of the contralateral nerve trunk at $2.5 \times$ sensory threshold. (C) Recording in right ulnar nerve in response to a tone burst. (D) Recording in left ulnar nerve in response to skin stimulation via subcutaneous needles at $10 \mathrm{~Hz}$ for $300 \mathrm{~ms}$ (25V, I ms pulses).

A recording in which nerve trunk stimulation to the contralateral nerve was given every 5 seconds (fig 4) was divided into successive batches each containing 16 evoked responses. The latency to the onset and the duration of successive average responses appeared identical (fig 4). In addition, average responses that were useful required that the recordings used for analysis were much longer than any random fluctuations that might have occurred in skin sympathetic discharges. ${ }^{10}$ The periodicities of all the fluctuations in these discharges are unknown. The respiratory related periodicities in skin sympathetic activity were very much less than the duration of the seven recordings used in the analysis.

The design of the experiment was open to criticism because the patterned nature of the stimuli resulted in anticipatory changes in the level of arousal even with the subject visually and aurally isolated. Hence, stimuli were given randomly in time, the state of arousal being maintained by the rare coupling of a stimulus with the simultaneous delivery of a painful electric shock to the forehead. The evoked response which occurred when forehead shock was combined with another stimulus was not included in any average. Responses evoked by stimuli which were given randomly were checked for constant latencies by comparing the averages of the first and last 16 responses of a recording. The interstimulus interval for randomly given stimuli ranged from 1.5 to 26 (mean, 14) seconds and the responses to stimuli in which the previous interstimulus interval was less than 5 seconds were not included in an average. It appeared that the amplitude of successive averages remained more constant with random rather than with patterned stimuli.

\section{Nerve trunk stimulation}

The averaged evoked skin sympathetic response to the stimulation of the contralateral peripheral nerve at stimulus levels two to three times the sensory threshold was obtained on six occasions. Recordings were obtained from the median and ulnar nerve at both wrists and in two of the six averages stimuli were not given randomly (fig 5). As differences in amplitude of average responses have no meaning for between recording session comparisons, the maximum amplitudes were normalised. At different recording

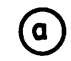

(a)

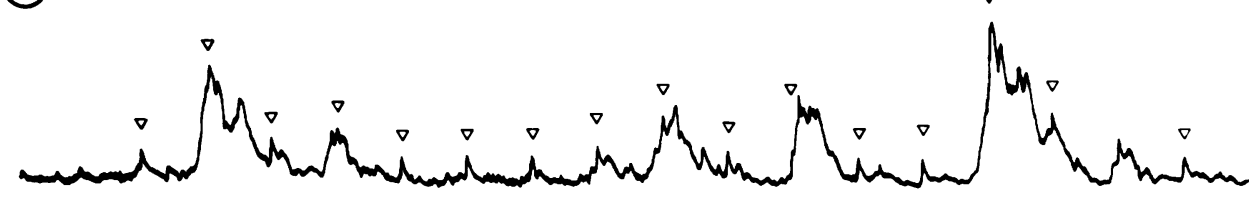

(b)

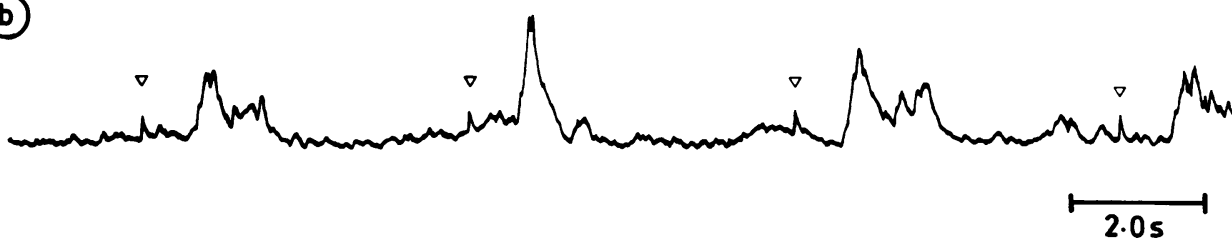

Fig 3 Bursts of skin sympathetic nerve activity in the left ulnar nerve evoked by stimulation of the contralateral ulnar nerve at $2.5 \times$ sensory threshold. Upper trace: stimulation at $1 \mathrm{~Hz}$. Lower trace: stimulation at $0 \cdot 2 \mathrm{~Hz}$. Infrequent stimuli were more predictably followed by bursts than frequent stimuli. 

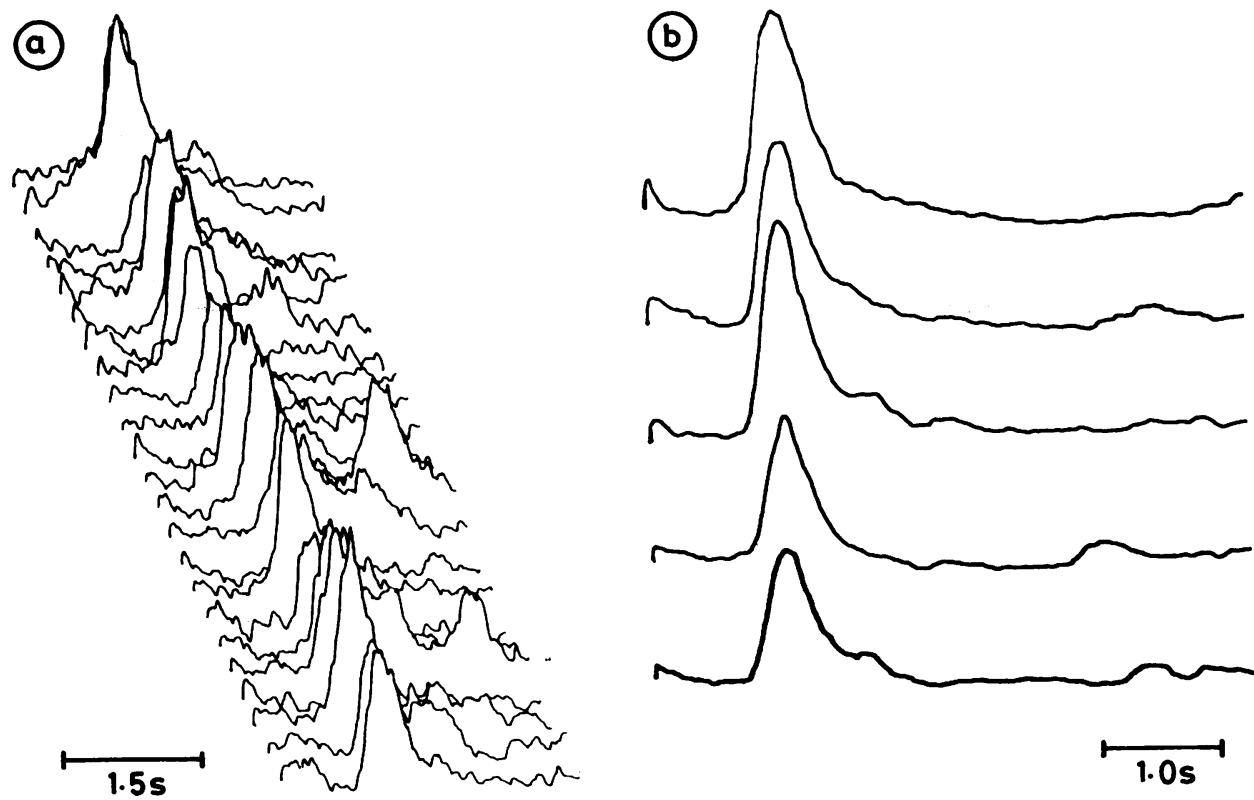

Fig 4 (A) Bursts of skin sympathetic nerve activity recorded in the left ulnar nerve and displayed as the integrated neurogram. The stimulus was nerve trunk stimulation to the contralateral ulnar nerve $(2.5 \times$ sensory threshold) every 5 seconds. The stimulus for each of the 23 responses occurred at the left of each trace and the successive responses are displayed from top to bottom. (B) Successive averages each containing sixteen responses. Note that the amplitude of successive averages declines in the situation where stimuli are given at fixed intervals.

sessions, latency varied from $540 \mathrm{~ms}$ to $680 \mathrm{~ms}$ (mean, 630; SD 50) in different nerves (fig 6). In some responses latency was difficult to measure because of the difficulty of identifying the take off of the response. The time to the half amplitude point was measured. The latency $(50 \%)$ ranged from 700 to $870 \mathrm{~ms}$ (mean, 780; SD 70).

The latency $(50 \%)$ for a single recording from the median nerve at the elbow was $550 \mathrm{~ms}$, the difference between this and the mean latency to the wrist being greater than three standard deviations of the mean latency at the wrist (fig 6). The distance between these recording sites was $220 \mathrm{~mm}$ which gave an approximate velocity of the fastest components of the evoked sympathetic response of $0.96 \mathrm{~m} / \mathrm{s}$.

When the different averages obtained at the wrists were compared, the reproducibility of the onset of the responses was greater than that of the later components (fig 5). Both monophasic and biphasic responses were obtained and all responses appeared complete within 2 seconds. Median and ulnar responses exhibited both mono and biphasic shapes.

Nerve trunk stimulation versus tone burst

The hypothesis that skin sympathetic responses to nerve trunk stimulation and noise are identical was tested by comparing the average evoked responses in a situation where both stimuli were delivered at random times in a random sequence. When the responses were compared, they were similar as far as latency, duration and shape were concerned (fig 7). The amplitude of the response to auditory tone bursts appeared to be less. A similar result was obtained when the responses to an auditory tone burst and skin stimulation (subcutaneous needles 2 to $3 \mathrm{~cm}$ apart; $10 \mathrm{~Hz}$ for $300 \mathrm{~ms} ; 25 \mathrm{~V} ; 1 \mathrm{~ms}$ ) were compared. It was concluded that the responses of skin sympathetic nerves to arousal stimuli of different types were temporally the same.

\section{Discussion}

The present study demonstrated that a variety of arousal stimuli produced a similar temporal activation of skin sympathetic nerve fibres. Comparisons of this type were carried out under particular experimental conditions, the essential features of which were control of the arousal state of the subject, the random delivery of stimuli both in time and of type, and the necessity for using long interstimulus intervals. In establishing the legitimacy of averaging evoked skin sympathetic responses, the present study 

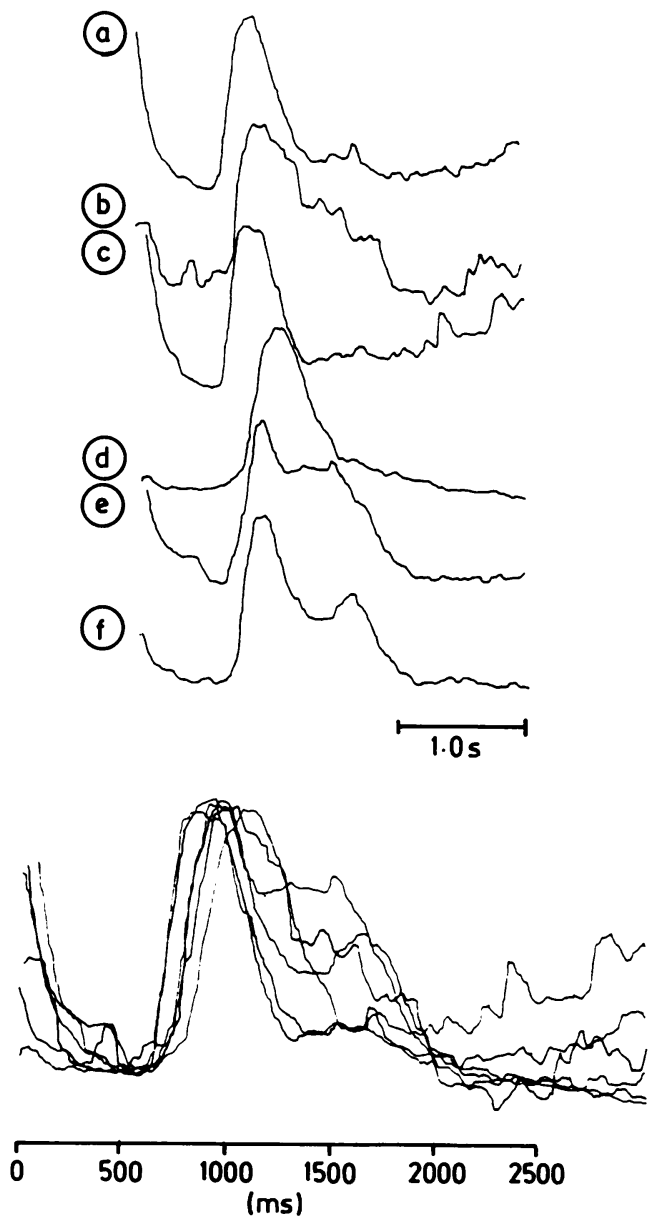

Fig 5 Average evoked skin sympathetic responses obtained from six recordings of skin sympathetic nerve activity in ulnar and median nerves at the level of the wrist. Median nerve recordings $A$ and $C$. Non random stimulation of the contralateral nerve in $D$ and $F$. The maximum amplitude of the responses was normalised in $G$, in which all traces $(A-F)$ are superimposed to facilitate comparison.

has shown that arousal induced responses have reproducible durations as well as latencies and that the shape and amplitude of arousal responses are the parameters that predominantly vary between recordings in a single subject.

The nerve activity recorded in the present study was impulses in sudomotor and vasomotor unmyelinated fibres. It has been considered sufficient in recent studies to rely solely on the characteristics of the discharge pattern rather than use local blockade and double nerve recordings to prove the unmyelinated efferent nature of the nerve fibres. ${ }^{3411}$ The single estimation of conduction velocity in the

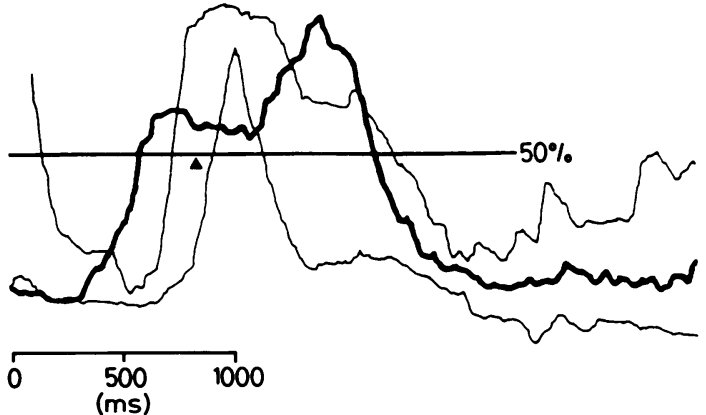

Fig 6 Comparison of an average skin sympathetic evoked response obtained from the median nerve at the elbow (dark line) with the range of all the average evoked responses obtained in median and ulnar nerves at the level of the wrist (range of responses lie between light lines; see fig 5). The mean latency to the half amplitude point for all wrist recordings is shown by arrow.
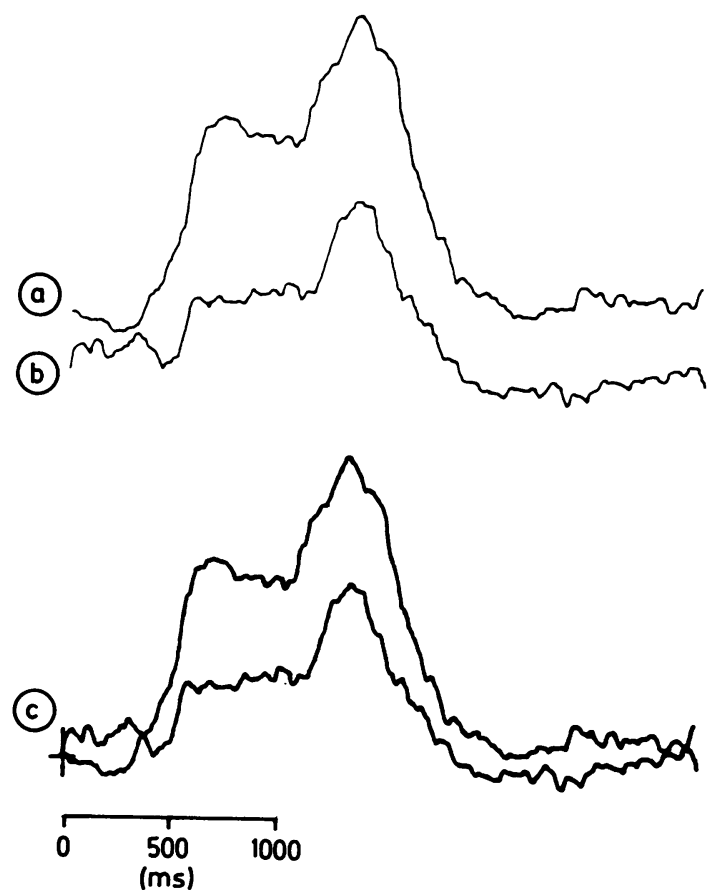

Fig 7 A comparison of an average skin sympathetic response evoked by contralateral nerve trunk stimulation $(A)$ with an average response evoked by a tone burst $(B)$. The similar shapes, latencies and duration are evident in $(C)$, which also permits a direct comparison of the amplitudes.

present study suggested that recordings were obtained from unmyelinated efferent fibres. Individual bursts of skin sympathetic nerve activity in response to nerve trunk stimulation or to a tone burst were identical to those described in previous work. ${ }^{13-511}$ 
Although the results in the present study were only obtained in a single subject, the conclusions reached about the methods that should be employed to obtain averages of evoked sympathetic responses, the properties of such averages and the factors affecting these responses were of general relevance. Some of the features of these methods, namely the importance of random stimuli and long interstimulus intervals have been noted as being essential prerequisites for quantifying peripheral autonomic surface potentials in man. ${ }^{12}$ In this discussion, it is worthwhile considering the latency, amplitude, pattern, duration and post response period of the evoked sympathetic response as distinct entities.

A variation in latency with subject height and extremity length has been described before. ${ }^{5}$ When allowances were made for these influences and the fact that recordings were obtained at the wrist rather than at the elbow, the average latency and the range of latencies within a particular recording session were very similar in the present and previous study. However, in the present work there was a variation in the mean latency between recordings of $170 \mathrm{~ms}$ compared with a variation of $10 \mathrm{~ms}$ in three subjects studied twice in the previous work. It is possible that some of this variation might have been due to changes in the experimental protocol which occurred as the method was developed. However, these changes, the introduction of random stimulation and control of the arousal state of the subject appeared to be more important for maintaining the amplitude of an average response rather than altering the temporal properties. Recordings were obtained from different nerves but no consistent trend was observed when the latencies of the evoked responses in the ulnar versus the median nerve of the wrist were compared. Other factors were probably more important. In the present study, recordings were obtained over a period of many months in a recording room in which environmental conditions fluctuated significantly. While temperature dependant changes in conduction velocity in unmyelinated fibres may have had a minor effect, variations in the nerve fibres activated in the responses, with consequent changes in conduction velocity were probably important. Skin sympathetic bursts comprising only vasoconstrictor impulses have lower conduction velocities than bursts containing only sudomotor impulses. ${ }^{5}$ Moderate warming makes the composition of arousal evoked responses exclusively sudomotor while moderate cooling shifts the composition to slower conducting impulses in vasoconstrictor fibres. ${ }^{3}$ Thus, the latency variation between studies may have reflected thermo-regulatory control systems engaging different groups of preganglionic and hence postganglionic neurons. Conduction velocity differences in the different nerve fibres could have caused the latency variations. These differences would be enhanced by the length of postganglionic axons and their relatively slow velocities.

Factors which caused variation in the pattern and amplitude of the responses would have included all those that affected the latency of the responses. As it is likely that the proportion of sudomotor to vasomotor activity was not constant because of changing environmental conditions, variations in the pattern of the average evoked response was not surprising. While all of these factors probably affected the amplitude of the average responses other technical factors were probably more important. Signal size in a microneuronographic recording depends upon a variety of factors some of which are unrelated to the amount of activity in sympathetic postganglionic fibres; these are related to the electrode impedance in situ and the position of the electrode tip in relation to the fibres of interest. Further, a noise reducing circuit is used routinely in these recordings and its setting critically affects signal and average response amplitude. Thus, amplitude comparisons normally have little meaning. It would appear that the only time the amplitudes of arousal responses could be compared, was when two stimuli were given in the one recording session. Even comparisons of the amplitudes of averages between successive recording sessions would seem to be inadvisable.

The present study demonstrated that the duration of an averaged evoked response was about $1500 \mathrm{~ms}$. In some averaged responses it appeared as if there was a period immediately after the response when some inhibition of background activity may have occurred (fig 7). This observation would be consistent with the suppression of skin sympathetic activity that has been observed in this situation during the rare recordings of activity in a single or a few sympathetic efferent fibres. ${ }^{7}$ In some responses, but not all, the nadir of the average corresponded to a period when the variance of the average was least.

The demonstration in the present study that a variety of arousal stimuli produce a similar temporal activation of skin sympathetic nerve fibres confirms the preliminary observations made by others. ${ }^{12}$ The precision concerning onset and duration of arousal induced responses that is provided by the microneuronographic technique might not be sufficient to detect the small difference in the afferent transmission times between nerve trunk stimulation and tone bursts. The central processing of these stimuli and their afferent conduction times take up only about 15 to $20 \%$ of the total latency period when skin sympathetic recordings are obtained at the wrist. Thus, it will be difficult to make any observation on interacting inputs onto sympathetic premotor neurons with the present method. 
However, the techniques and results described in the present study are useful for furthering our understanding of other properties of premotor sympathetic, sympathetic preganglionic neurons and their interconnecting pathways. It has been observed by others that under conditions in which skin sympathetic activity is high successive sudomotor bursts occur at preferred intervals which are integral multiples of a period of about $0.6 \mathrm{~s}^{3}$ The same applies to the duration of intervals between vasoconstrictor bursts. Some of the averaged evoked responses in the present study were definitely biphasic, the period between peaks in these averages being 620,550 and $610 \mathrm{~ms}$ (fig $5 F$ ). Identification of the factors that produce biphasic responses and which can alter these basic periodicities will be facilitated by the use of the methods described in the present study.

This project was initiated by Sir Roger Bannister, who also provided continuous encouragement. The work was supported by a grant from The Medical Foundation, The University of Sydney. PM Satchell received a Thomas and Ethel Mary Ewing Scholarship. Professor TA Sears provided invaluable help during the project. Professor JC McLeod, Dr M Hersch, Mr C Vaughan and Ms P Sparkes helped greatly with the manuscript.

\section{References}

1 Hagbarth KE, Hallin RG, Hongell A, Torbjork HE, Wallin BG. General characteristics of sympathetic activity in human skin nerves. Acta Physiol Scand 1972;84:164-76.

2 Delius W, Hagbarth KE, Hongell A, Wallin BG. Manoeuvres affecting sympathetic outflow in human skin nerves. Acta Physiol Scand 1972;84:177-86.

3 Bini G, Hagbarth KE, Hynninen P, Wallin BG. Thermoregulatory and rhythm generating mechanisms governing the sudomotor and vasoconstrictor outflow in human cutaneous nerves. J Physiol (Lond) 1980; 306:537-52.

4 Bini G, Hagbarth KE, Hynninen P, Wallin B. Regional similarities and differences in thermoregulatory vasoand sudomotor tone. $J$ Physiol (Lond) 1980;306: 553-65.

5 Fagius J, Wallin BG. Sympathetic reflex latencies and conduction velocities in normal man. $J$ Neurol Sci 1980;47:433-48.

6 Wallin BG, Stjernberg L. Sympathetic activity in man after spinal cord injury. Brain 1985;107:183-98.

7 Hallin RG, Torebjork HE. Single unit sympathetic activity in human skin nerves during rest and various manoeuvres. Acta Physiol Scand 1974;92:303-17.

8 McKeon B, Burke D. Identification of muscle spindle afferents during in vivo recordings in man. Electroencephalogr Clin Neurophysiol 1980;48:606-8.

9 Shahani BT, Halperin JJ, Boulu P, Cohen J. Sympathetic skin response-a method of assessing unmyelinated axon dysfunction in peripheral neuropathies. $J$ Neurol Neurosurg Psychiatry 1984;47:536-42.

10 Bendat JS, Piersol AG. Measurement and Analysis of Random Data. New York: Wiley, 1982:219-23.

11 Normell LA, Wallin BG. Sympathetic skin nerve activity and skin temperature changes in man. Acta Physiol Scand 1974;91:417-26.

12 Knezevic W, Bajada S. Peripheral autonomic surface potential. A quantitative technique for recording sympathetic conduction in man. J Neurol Sci 1985; 67:239-51. 